\title{
Image Quality Assessment Method Based on Contourlet Transform and Generalized Gamma Distribution
}

\author{
Bin Wang ${ }^{1, a^{*}}$ \\ ${ }^{1}$ Department of Mechanical and Electronic Engineering, Jiaxing University, Jiaxing, Zhejiang \\ Province, China \\ aiseewb@gmail.com
}

\begin{abstract}
Keywords: Image Quality Assessment; Contourlet Transform; Generalized Gamma Distribution. Abstract. This paper proposes a new image quality assessment approach based on Contourlet transform and generalized gamma distribution (GGD). The image is processed by Contourlet transform at first and the Contouelet coefficients are modeled by GGD. The parameter of GGD is estimated and the feature vector is formed by combining the parameter of GGD. The feature vector is fed to the support vector machine for training and testing. Experimental results on LIVE database show that our approach has good correlation with the subjective character of human vision.
\end{abstract}

\section{Introduction}

The image quality assessment plays an important role in image/video processing area. Image quality assessment approach can be divided into full-reference (FR), reduced-reference (RR) and no-reference (NR) methods according to the availability of a reference image[1]. There are many good approaches about FR and RR. However, there are many rooms of improvement for the NR image quality assessment method. The NR image quality assessment approach can be divided into natural scene statistics approaches and training-based approaches[1]. Natural scene statistics approaches uses probability density function such as general Gaussian distribution and general gamma distribution to model the histogram of wavelet coefficients or DCT (discrete cosine transform) coefficients of the image[3]. The training-based approach extracts feature vectors from the image and use support vector regression (SVR) to estimate the quality score of the image[4].

This paper proposes a new NR image quality assessment method based on the Contourlet Transform (CT). The method performs Contourlet Transform on the image to get Contourlet coefficients for different order. After that, histograms of Contourlet coefficients are modeled by different generalized gaussian distributions. Then parameters of these generalized gaussian distributions are used to form the feature vector. Finally, the feature vector is fed to support vector regression machine to get the score of the image.

\section{Contourlet Transform and Generalized Gamma Distribution}

Contourlet transform is proposed by Minh. N. Do and Martin Vetterli in 2005[1], it is a kind of sparse representation of the signal. The contourlet transform combines the Laplacian Pyramid(LP) and the directional filter banks(DFB) together. When applying for the image, the contourlet transform performs the Laplace pyramid decomposition on the image for multi-resolution decomposition at first, and then performs multi-direction filter bank on the high-frequency band of Laplace decomposition. The flow of Contourlet transform is shown in figure 1[1]. LP decomposes the image into low-pass subbands and high-pass subbands, and DFB decomposes the high-pass subbands further into subbands in different directions. In this way, the image is decomposed into subbands in different scales and different directions.

The generalized Gaussian distribution is used to model the histogram of DCT coefficients of the image in [6]. The generalized gamma distribution includes the Gamma, Gaussian and Laplace distribution as special cases[7]. According to [7], the experiments show that for most of images, the statistics of the DCT coefficients can be better approximated by generalized gamma distribution than generalized Gaussian distribution. According to [8] and [9], the histogram of wavelet coefficients of the image can not be well modeled by generalized Gaussian distribution since the peak of the 
histogram is often shifted left or right toward zero. The generalized gamma distribution has one more parameter than generalized Gaussian distribution which is named index shape parameter, and the index shape parameter provides more flexibility for the generalized gamma distribution than the generalized Gaussian distribution. In this paper, we also use the generalized gamma distribution to model the Contourlet coefficients of the image. The Contourlet coefficients in one orientation of $8 \times 8$ block are combined together and modeled by generalized gamma distribution.

The probability density function of generalized gamma distribution is defined as[10]:

$$
p(x)=\frac{\beta x^{\beta \lambda-1}}{2 \alpha^{\beta \lambda} \Gamma(\lambda)} \exp \left(-\left(\frac{|x|}{\alpha}\right)^{\beta}\right)
$$

In which: $\alpha$ is the scale parameter, $\beta$ is the shape parameter, $\lambda$ is the index shape parameter. $\Gamma(\cdot)$ is the gamma function which is defined by: $\Gamma(\lambda)=\int_{0}^{\infty} x^{\lambda-1} e^{-x} d x$ for $\lambda>0$.

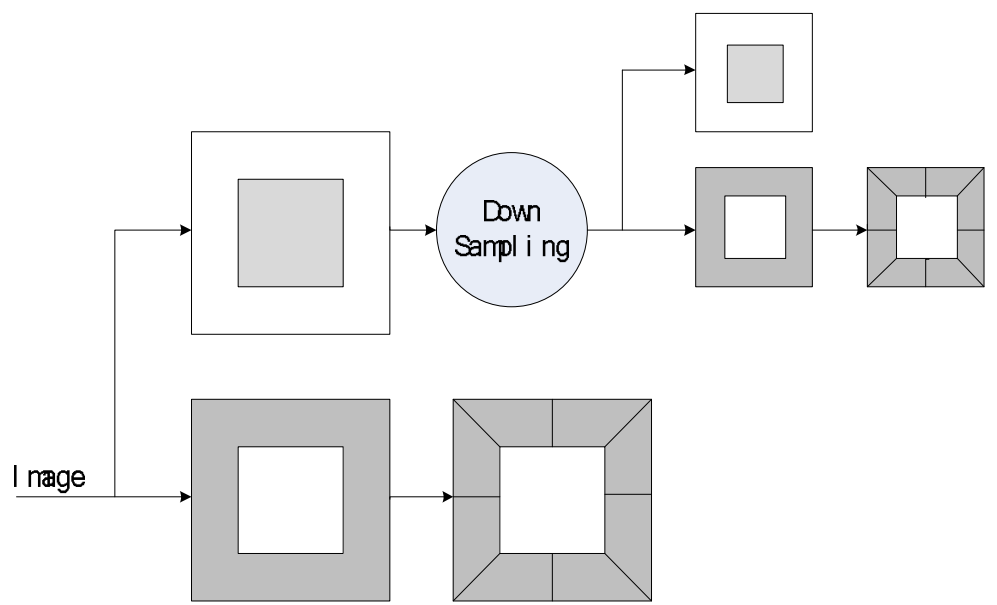

Fig.1. Flow of Contourlet Transform

In order to estimate the scale parameter, shape parameter and index shape parameter, we use the method proposed in [9] and [11]. For the sequence of Contourlet coefficients denoted by $x=\left\{x_{1}, x_{2}, \ldots, x_{N}\right\}$, the maximum likelihood estimation approach is used to estimate the parameter. If the statistics is given by[7]:

$$
\begin{gathered}
S_{1}(\beta)=\frac{1}{N} \sum_{k=1}^{N}\left|x_{k}\right|^{\beta} \\
S_{2}(\beta)=\frac{1}{N} \sum_{k=1}^{N}\left|x_{k}\right|^{2 \beta} \\
S_{3}(\beta)=\frac{1}{N} \sum_{k=1}^{N} \log \left|x_{k}\right|^{\beta} \\
S_{4}(\beta)=\sum_{k=1}^{N}\left|x_{k}\right|^{\beta} \log \left|x_{k}\right| \\
S_{5}(\beta)=\frac{1}{N} \sum_{k=1}^{N} \log \left|x_{k}\right|
\end{gathered}
$$

The shape parameter $\beta$ can be estimated by the following scale-independent shape estimation (SISE) equation:

$$
\phi(\beta)=\log S_{2}(\beta)-2 \log S_{1}(\beta)-\log \left[1+\beta\left(\frac{S_{4}(\beta)}{S_{1}(\beta)}-S_{5}(\beta)\right)\right]=0
$$


The Newton-Raphason method is used for iterative computation of the shape parameter $\beta$ as follows:

$$
\hat{\beta}^{(n+1)}=\hat{\beta}^{(n)}-\mu \frac{\phi\left(\hat{\beta}^{(n)}\right)}{\phi^{\prime}\left(\hat{\beta}^{(n)}\right)}
$$

\section{Flow of Our Approach}

The framework of our approach is shown in Fig. 1. The image set is divided into training images and testing images. Since all image in the image set is color image, the color image is transformed into gray image at first, and then the gray image is subjected to the Contourlet transform $(\mathrm{CT})$ module to compute the CT coefficients of the image. After that, the CT coefficients at different scales and different directions are modeled by generalized gamma distribution, and the parameter estimation method is used to estimate parameters of generalized gamma distribution. At last, those parameters are combined into a feature vector, and the vector is subjected to the support vector regression (SVR) machine for trainning and testing for get the quality score of the input image.

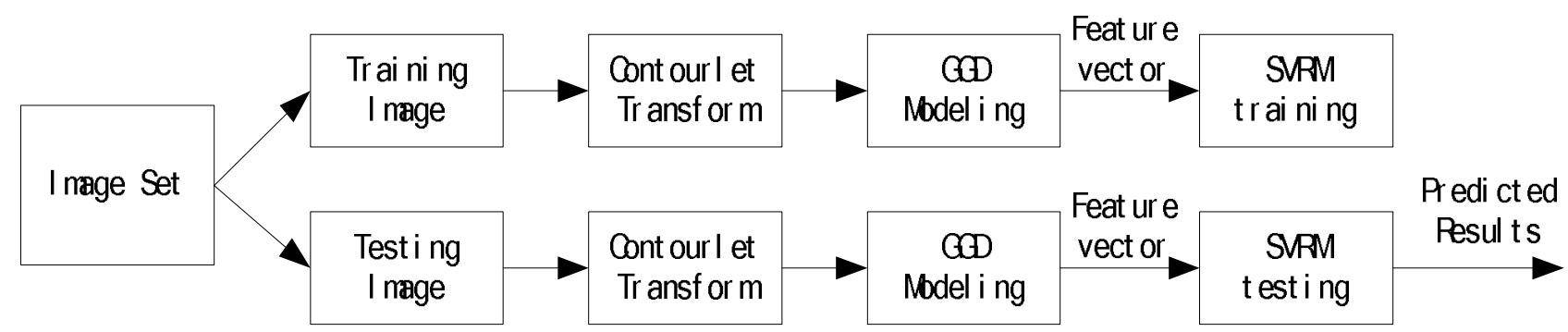

Fig.2. Flowchart of Contourlet-based Image Quality Assessment Approach

In the above flow, the feature vector of the image are formed by combining the shape parameter, scale parameter and the index shape parameter of the Contourlet coefficients. The image set can be divided into five categories, which are JPEG, JPEG2000, white noise (WN), Gaussian blur (GB) and fast-fading (FF) channel distortion [6]. Before the classification, the SVM is trained by using the training set of the distorted image. After training the support vector regression machine, the feature vector is subjected to the support vector regression machines to predict the quality score of the image.

\section{Experimental Results}

The LIVE image quality assessment database [6] is used in the experiments. The order of fractional Fourier transform is $0.25,0.5,0.75$ and 1 . The image is divided into $8 \times 8$ blocks. There are 29 reference images in the LIVE IQA database, and there are 779 distorted images. We divide 29 reference images into two parts: 20 images are used for training, and the remaining 9 images are used for testing.

The LIVE (Laboratory for Image and Video Engineering) image quality assessment database [3, 6, 7] is used to evaluate the performance of our approach. The LIVE IQA database has 29 reference image and 779 distorted image with five types, which are JPEG2000 Compression (JPEG2K), JPEG Compression (JPEG), White Noise (WN), Gaussian Blur (GB) and Fast Fading (FF). he LIVE IQA database is divided into two parts: training image and testing image. The degraded version of 24 reference images are used for training, and the degraded version of remaining 5 reference images are used for testing. 
The Spearman rank order correlation coefficient (SRCC) and Pearson linear correlation coefficient (PLCC) are used to evaluate the performance of different algorithms. Two other approaches which are PSNR and MS-SSIM are used to compare with our approach. The detailed result is shown in Table I. According to table I, the proposed approach is superior than the PSNR approach by evaluating the SRCC and PLCC coefficients between the distorted image and the reference image in testing image database.

Table I. Spearman Rank Order Correlation Coefficient of Different Algorithm (aligned DMOS)

\begin{tabular}{|l|l|l|l|l|l|l|}
\hline & JP2K & JPEG & WN & GB & FF & ALL \\
\hline PSNR & 0.8954 & 0.8809 & 0.9854 & 0.7823 & 0.8907 & 0.8755 \\
\hline SSIM & 0.9613 & 0.9754 & 0.9748 & 0.9474 & 0.9537 & 0.9478 \\
\hline CBIQ & 0.8935 & 0.9418 & 0.9582 & 0.9184 & 0.8856 & 0.8967 \\
\hline VIF & 0.9322 & 0.9533 & 0.9531 & 0.9433 & 0.9532 & 0.9472 \\
\hline Proposed & 0.9357 & 0.9546 & 0.9632 & 0.9435 & 0.9573 & 0.9568 \\
\hline
\end{tabular}

Table II. Pearson Correlation Coefficient of Different Algorithm (aligned DMOS)

\begin{tabular}{|l|l|l|l|l|l|l|}
\hline & JP2K & JPEG & WN & GB & FF & ALL \\
\hline PSNR & 0.8769 & 0.8674 & 0.9294 & 0.7746 & 0.8760 & 0.8591 \\
\hline SSIM & 0.8845 & 0.9268 & 0.9560 & 0.8820 & 0.9349 & 0.8959 \\
\hline CBIQ & 0.8935 & 0.9217 & 0.9281 & 0.9215 & 0.8722 & 0.9077 \\
\hline VIF & 0.8833 & 0.9425 & 0.9326 & 0.9325 & 0.8532 & 0.8942 \\
\hline Proposed & 0.9042 & 0.9321 & 0.9532 & 0.8702 & 0.8421 & 0.9062 \\
\hline
\end{tabular}

\section{Conclusions}

In this paper, the Contourlet transform and the generalized gamma model are used to predict the quality score of the distorted image. The Contourlet coefficients are modled by the generalized gamma distribution, and parameters of generalized gamma distribution are combined as the feature vector. The feature vector is send to the support vector regression machine for training and testing. The experiment result of LIVE IQA database shows that our approach can compete with the BLIINDS-II algorithm in evaluating the quality score of the image.

\section{References}

[1] M. N. Do and M. Vetterli, The Contourlet Transform: An Efficient Directional Multiresolution Image Representation, IEEE Transaction on Image Processing, 14(2005) 2091-2103.

[2] P. Ye, D. Doermann, No-Reference Image Quality Assessment using Visual Codebooks, IEEE Transaction on Image Processing, 21(2012) 3129-3138.

[3]. A. K. Moorthy, A. C. Bovik, A Two-step Framework for Constructing Blind Image Quality Indices, IEEE Signal Processing Letters, 17(2010) 513-516.

[4] H. Tang, N. Joshi, A. Kapoor, Learning a Blind Measure of Perceptual Image Quality, IEEE Conference on Computer Vision and Pattern Recognition, (2011) 305-312.

[5] M. A. Saad, A. C. Bovik, Christophe Charrier, Model-Based Blind Image Quality Assessment Using Natural DCT Statistics, IEEE Transaction on Image Processing, 21(2011) 3339-3352. 